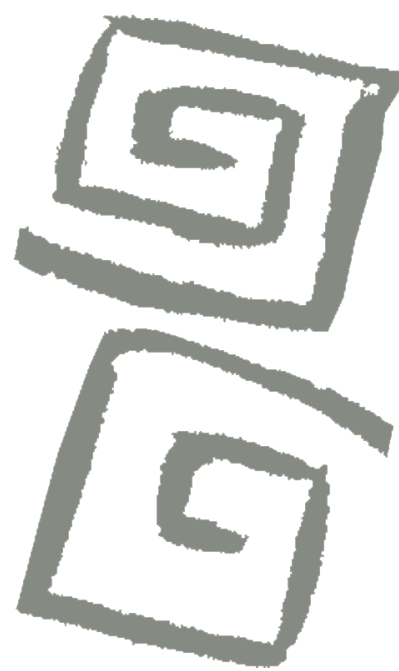

\title{
Reducción de la cobertura social para los fármacos antiartrósicos sintomáticos de acción lenta: una iniciativa de desinversión en Argentina, 2015-2017
}

\author{
Reduction of social coverage for symptomatic \\ slow-acting drugs for osteoarthritis: a disinvestment \\ initiative in Argentina, 2015-2017
}

${ }^{1}$ Autor de correspondencia. Médico, Magíster en Epidemiología, Gestión y Políticas de Salud. Área Farmacología, Fundación FEMEBA, La Plata, Argentina. Instituto de Ciencias de la Salud, Universidad Nacional Arturo Jauretche, Florencio Varela, Argentina. $\triangle$ (iD)

${ }^{2}$ Médica Clínica. Gerencia de Medicamentos, INSSIP, Ciudad Autónoma de Buenos Aires, Argentina. $\square$ (iD

${ }^{3}$ Médico, Magíster en Farmacoepidemiología. Área Farmacología, Fundación FEMEBA, La Plata, Argentina. Instituto de Ciencias de la Salud, Universidad Nacional Arturo Jauretche, Florencio Varela, Argentina. $\triangle$ (iD)

${ }^{4}$ Médico Psiquiatra. INSSIP, Ciudad Autónoma de Buenos Aires, Argentina. Instituto de Neurología Cognitiva, Ciudad Autónoma de Buenos Aires, Argentina. $₫$ iD

${ }^{5}$ Doctor en Medicina. INSSJP, Ciudad Autónoma de Buenos Aires, Argentina. $\square$ (iD)

${ }^{6}$ Ingeniero en Sistemas de Información, Magíster en Administración. INSSJP Ciudad Autónoma de Buenos Aires, Argentina. $\square$ (iD)
Martín A. Urtasun', María Noble², Martín Cañás³, Julián Bustin4, Ricardo C. Mastai ${ }^{5}$, Alejandro J. Regueiro ${ }^{6}$

RESUMEN En abril de 2016, el Instituto Nacional de Servicios Sociales para Jubilados y Pensionados excluyó del subsidio social la cobertura al 100\% de 159 fármacos, entre ellos, los antiartrósicos sintomáticos de acción lenta o symptomatic slow-acting drugs for osteoarthritis (SySADOA), por insuficiente evidencia de beneficio clínico significativo. Evaluamos el efecto de esta medida sobre la utilización de SySADOA y de los antiinflamatorios no esteroides (AINE), no afectados por la medida. Se compararon las dispensas ambulatorias de los SySADOA y los AINE de 2015 a 2017, midiendo unidades dispensadas, precio de venta al público y gasto de bolsillo del beneficiario para cada mes. Luego de la medida, descendieron un 61,6\% los envases de SySADOA dispensados y un 63,4\% el monto total del precio de venta al público, medido en valores constantes. La dispensa no se reorientó hacia los AINE, que descendieron un 6,1\%. Disminuyó tanto la incidencia de nuevos tratamientos (de 6,4 a 3,3 tratamientos por 1.000 beneficiarios por mes) como su continuidad. El gasto de bolsillo de los beneficiarios en SySADOA aumentó un 75,8\% (a valores constantes). La desinversión en intervenciones de valor terapéutico cuestionable es una herramienta valiosa para la sustentabilidad de los sistemas de salud.

PALABRAS CLAVES Inversiones en Salud; Osteoartritis; Tratamiento Farmacológico; Glucosamina; Sulfatos de Condroitina; Antiinflamatorios no Esteroideos; Cobertura de los Servicios de Salud; Argentina.

ABSTRACT In April 2016, the National Institute of Social Services for Retirees and Pensioners discontinued its policy of $100 \%$ coverage for 159 drugs (the "social subsidy"), including symptomatic slow-acting drugs for osteoarthritis (SYSADOAs), due to insufficient evidence of significant clinical benefit. We evaluated the effect of this measure on the use of SYSADOAs as well as non-steroidal anti-inflammatory drugs (NSAIDs), which were unaffected by this policy change. We compared outpatient dispensations of SYSADOAs and NSAIDs from 2015 to 2017, measuring dispensed units, retail price, and out-of-pocket expenses for beneficiaries each month. After the change in coverage, there was a $61.6 \%$ total decrease in SYSADOA units dispensed, and a $63.4 \%$ decrease in the final sales price to the public, measured in constant values. Dispensation was not reoriented towards NSAIDs, which fell by $6.1 \%$. The incidence of new treatments decreased (from 6.4 to 3.3 treatments per 1,000 beneficiaries per month), as did their continuity. Beneficiaries' out-ofpocket spending on SYSADOAs increased by $75.8 \%$ (at constant values). Disinvestment in interventions with questionable therapeutic value is an important tool in working toward the sustainability of health systems.

KEY WORDS Investments; Osteoarthritis; Drug Therapy; Glucosamine; Chondroitin Sulfates; Anti-Inflammatory Agents, Non-Steroidal; Health Services Coverage; Argentina. 


\section{INTRODUCCIÓN}

Se denominan fármacos antiartrósicos sintomáticos de acción lenta o symptomatic slowacting drugs for osteoarthritis (SySADOA) a un conjunto de medicamentos para la artrosis cuyo efecto clínico se produce luego de varias semanas de iniciado el tratamiento, en contraste con la rápida respuesta analgésica que logran el paracetamol y los antiinflamatorios no esteroides (AINE) ${ }^{(1)}$. Los SySADOA comercializados en Argentina incluyen condroitín sulfato, diacereína, glucosamina e insaponificables de palta y soja, y sus combinaciones ${ }^{(2)}$.

Su eficacia real como analgésicos y como modificadores del progreso de la enfermedad es controversial ${ }^{(1,3,4)}$. Los ensayos clínicos y las revisiones sistemáticas encuentran un efecto analgésico entre modesto y nulo, y una diferencia en la preservación del grosor del cartílago articular de la rodilla, cuya relevancia clínica es cuestionable ${ }^{(5,6,7,7,8,9,10)}$.

Las dudas sobre la eficacia de los SySADOA se reflejan en la disparidad de su cobertura por los sistemas públicos de salud. Así, por ejemplo, están financiados en España, pero no en Francia, Dinamarca y Suecia; en el Reino Unido y en EEUU se consideran suplementos dietarios y no tienen, por lo tanto, cobertura como medicamentos ${ }^{(1,11)}$. En Argentina, los SySADOA no están incluidos en el listado de medicamentos del Programa Médico Obligatorio ${ }^{(12)}$, pero tienen una cobertura del $50 \%$ para los afiliados del Instituto Nacional de Servicios Sociales para Jubilados y Pensionados (INSSJP) ${ }^{(2)}$. Esta institución estatal argentina organiza y financia la atención médica de los adultos mayores y de sus familiares a cargo, así como de personas de cualquier edad con pensiones por discapacidad y veteranos de guerra. Es más conocido como PAMI, por las siglas de Programa de Asistencia Médica Integral, y cubre a unos cinco millones de beneficiarios ${ }^{(13)}$. EI INSSJP financia el uso de medicamentos para pacientes ambulatorios con un porcentaje de descuento sobre el precio de venta al público ${ }^{(14)}$. Como en Argentina se comercializan diversas marcas de "medicamentos similares", es decir, aquellos con la misma composición, pero diferente fabricante y precio, el gasto de bolsillo del paciente dependerá de la marca elegida ${ }^{(15)}$.

Para los beneficiarios que por razones sociales no pueden pagar sus medicamentos ambulatorios con el descuento previsto, se estableció en 2005 un subsidio social que cubre el $100 \%$ del costo del medicamento ${ }^{(16)}$. El programa de subsidios sociales se extendió en los diez años siguientes, hasta cubrir cerca del $50 \%$ de las unidades de medicamentos dispensadas en $2016^{(17)}$.

Tal como se lo formuló inicialmente, el subsidio social cubría todo tipo de medicamentos, incluyendo algunos productos de valor terapéutico potencial dudoso o nulo, presentes en el mercado argentino ${ }^{(18)}$. Aunque la autorización de un nuevo fármaco en el país prevé el análisis de su "eficacia e inocuidad", dicha evaluación se homologa en forma automática cuando el medicamento ya está aprobado por la Food and Drug Administration, la European Medicines Agency u otras agencias de referencia ${ }^{(19)}$, que es el mecanismo utilizado para la mayoría de los nuevos registros ${ }^{(20)}$. Sin embargo, persisten en el mercado numerosos medicamentos más antiguos, cuyo certificado se renueva en forma prácticamente automática cada cinco años, incluso cuando algunos han sido retirados en otros países por sus efectos adversos ${ }^{(21,22)}$. Muchos de estos productos son combinaciones de dosis fijas, sin fundamento bibliográfico apropiado ${ }^{(23,24)}$. Por otro lado, para las autorizaciones subrogadas a una agencia de referencia, la normativa no contempla que el fármaco deba retirarse si se cancela la autorización en el país de origen ${ }^{(19)}$.

Una revisión efectuada en conjunto con el Instituto de Efectividad Clínica y Sanitaria -una institución académica afiliada a la Universidad de Buenos Aires- encontró que algunos de los medicamentos cubiertos por este mecanismo correspondían a fármacos "cuya evidencia médica disponible no resulta suficiente para determinar beneficios clínicos significativos"(25). En consecuencia, el INSSJP dictó la Resolución 439/2016 que, si bien excluyó un listado de 159 monofármacos o 
combinaciones de dosis fijas de la cobertura al $100 \%$, en todas sus presentaciones comerciales, mantuvo para el mismo listado el porcentaje de cobertura original. Los SySADOA fueron incluidos en este listado, quedando a partir de entonces con el descuento básico del $50 \%$ sobre su precio de venta al público $^{(25)}$.

Esta medida es un ejemplo de lo que en la bibliografía sanitaria se ha denominado "desinversión", esto es, la retirada parcial o completa de la financiación colectiva para un determinado bien o servicio destinado al cuidado de la salud, que se considera que provee poca o ninguna ganancia de salud para su costo y no es, por lo tanto, una asignación eficiente de los recursos, con una visión explícita de reorientar los fondos a medidas con mayor impacto sanitario(26,27,28). La "desinversión pasiva" es el abandono espontáneo de las prácticas que caen en desuso o que son reemplazadas por otras; la "desinversión activa" consiste en revisar la cobertura de aqueIlas tecnologías que han quedado obsoletas o no fueron correctamente evaluadas en el momento de su adopción ${ }^{(28)}$.

En este contexto, el incremento del gasto en medicamentos es una preocupación central para los financiadores públicos o privados que deben proveer atención médica integral y equitativa a partir de presupuestos sustentables. Para poder afrontar la permanente incorporación de fármacos de alto costo se promueve la revisión de la cobertura de tratamientos de bajo valor ${ }^{(26,28)}$. Las estrategias de desinversión incluyen diferentes alternativas: la reducción o suspensión total de la financiación colectiva (esta última medida denominada "delisting" en la bibliografía en inglés), la incorporación de reglas de uso para determinados fármacos, la selección de algunos principios activos dentro de cada clase terapéutica y la promoción de la prescripción de medicamentos genéricos ${ }^{(26,28)}$.

Las intervenciones de desinversión tienen consecuencias para todas las partes involucradas, incluyendo los pacientes, los prescriptores, los hospitales, los fabricantes y los financiadores. Sus efectos incluyen aspectos clínicos, económicos y de satisfacción de los diversos actores. La evaluación de los resultados de una intervención de este tipo contribuye a comprender sus consecuencias e informar decisiones futuras ${ }^{(28,29)}$.

El análisis de experiencias de desinversión en medicamentos por parte de los sistemas públicos de salud de España ${ }^{(30,31)}$, Francia ${ }^{(29,32,33)}$, Irlanda ${ }^{(34)}$ y en un conjunto de seis países de la Organización de Cooperación y Desarrollo Económico ${ }^{(28)}$ muestra algunas constantes. La primera es un rápido descenso de la prescripción y dispensa de los fármacos cuya cobertura se redujo, con la consiguiente caída del gasto por parte del Estado, y un aumento del gasto de bolsillo para los pacientes que continúan usando estos medicamentos ${ }^{(30,31,32)}$.

Un segundo aspecto destacado es la necesidad de evaluar si la reducción de cobertura a unos medicamentos reorienta el consumo hacia otros que la conservan o, eventualmente, si se aumenta la demanda de otros servicios de salud, como podrían ser las consultas ${ }^{(35)}$. Por ejemplo, al retirarse en Francia la financiación para los medicamentos mucolíticos aumentó el consumo de antitusivos y broncodilatadores $^{(29)}$. Por otra parte, dada la existencia de distintas presentaciones comerciales con igual composición, podría esperarse que quienes sigan utilizando el fármaco se inclinen por los productos de menor precio. El resultado final puede no ser el buscado: en una revisión de intervenciones de desfinanciación, Chambers et al. encuentran que, en una quinta parte de las experiencias, los gastos totales en realidad aumentaron $^{(36)}$.

Otros componentes importantes a incluir en una evaluación integral de estas intervenciones son los resultados de salud, económicos y de satisfacción desde los puntos de vista de los pacientes, los prestadores y el financiador ${ }^{(28,33,36,37)}$. Esta evaluación completa es de difícil cumplimiento, y las experiencias publicadas muestran un compromiso entre lo ideal y lo factible ${ }^{(27)}$.

El presente trabajo evaluó los efectos sobre la dispensa y el gasto de excluir del subsidio social del INSSJP la cobertura del 100\% en medicamentos SySADOA, y exploró las alternativas de tratamiento farmacológico utilizadas a partir de esa medida. 


\section{MÉTODOS}

Se realizó un estudio de utilización de medicamentos observacional retrospectivo, que analiza la base de datos administrativa de dispensa ambulatoria de medicamentos para los beneficiarios del INSSJP durante el trienio 2015-2017. El cambio de cobertura entró en vigencia el 7/4/2016, por lo que se cuenta con datos de 15 meses previos y 20 meses posteriores a la medida.

Entre los 159 principios activos o combinaciones de dosis fijas excluidos de la cobertura del 100\% por la Resolución 439/2016 se identificaron los SySADOA, ubicados en el capítulo M01A ("Productos antiinflamatorios y antirreumáticos, no esteroides") del Sistema de Clasificación Anatómica, Terapéutica, Química -Anatomical Therapeutic Chemical Classification System (ATC)- de la Organización Mundial de la Salud ${ }^{(38)}$, los que se denominarán en adelante "fármacos A". Para explorar la eventual reorientación de la prescripción hacia fármacos no alcanzados por la limitación de la cobertura, se estudió la evolución de la dispensa de los restantes medicamentos del capítulo M01A y del analgésico paracetamol, que se denominarán "fármacos B". Se obtuvo el detalle de todas las presentaciones comerciales de los medicamentos seleccionados disponibles en Argentina, buscando los principios activos en la base de datos farmacéutica comercial AlfaBeta, que utiliza el INSSJP para clasificar los medicamentos $^{(2)}$. Se obtuvieron los datos mensuales del número de envases dispensados, precio de venta al público y monto efectivamente abonado por el beneficiario para todos los fármacos de las listas A y B.

Para evaluar la evolución del gasto en un contexto de acentuada inflación y debido a los cuestionamientos a las mediciones nacionales del Instituto Nacional de Estadística y Censos (INDEC) entre 2007 y $2015^{(39)}$, se ajustaron los valores a pesos de enero de 2015, utilizando los datos del Índice de precios al consumidor de la Ciudad Autónoma de Buenos Aires ${ }^{(40)}$.

Se calculó la evolución del porcentaje promedio del precio de venta al público cubierto por el INSSJP, el cual refleja la combinación entre beneficiarios que accedieron al subsidio social del $100 \%$ del precio de venta al público del medicamento y los que obtuvieron el descuento estándar del 50\%.

Se calculó la proporción de prevalencia de uso mensual (en adelante "prevalencia de uso") de los fármacos de cada lista como la proporción del total de beneficiarios a los que se les dispensaron medicamentos de esa lista durante el mes ${ }^{(41)}$. Los cambios en la prevalencia de uso pueden deberse tanto a modificaciones de la incidencia de nuevos tratamientos como a una variación en su duración. Para evaluar la contribución del primer componente se analizó la tasa de incidencia de nuevos usuarios, definidos como aquellos pacientes con dispensa de un fármaco de la lista A o B en un mes dado, que no hayan tenido dispensa de medicamentos de la lista respectiva en los seis meses previos. Para estudiar la continuidad posterior del tratamiento se analizó la dispensa del fármaco en los meses subsiguientes, calculando la proporción de pacientes que luego de iniciar un tratamiento nuevo recibían una segunda y una tercera dispensa, siempre que el seguimiento hubiera durado al menos cuatro meses desde la dispensa inicial.

Para resumir el efecto de la intervención se calcularon los promedios mensuales de las variables descriptas en tres períodos: uno previo a la intervención estudiada, desde enero de 2015 a marzo de 2016, una etapa de transición durante los primeros cuatro meses a partir de la medida, y un período posterior desde agosto de 2016 a diciembre de 2017.

A fin de caracterizar mejor el uso de los fármacos $\mathrm{A}$, se analizó la prevalencia de uso según el sexo y de acuerdo al lugar de residencia, definido según las 38 unidades de gestión local del INSSJP, comparando los resultados de un mes anterior a la medida (julio de 2015) con uno posterior (julio de 2017). Se exploró también si la indicación inicial de los fármacos A era realizada por el médico de cabecera del paciente o por un especialista, utilizando los datos administrativos disponibles en el sistema informático del INSSJP.

Por último, se evaluó si la pérdida del subsidio del $100 \%$ a los fármacos A generó una 
reorientación de la selección de marcas comerciales hacia productos más baratos con la misma composición. Con ese fin se tomó como caso testigo el fármaco A más utilizado (glucosamina + meloxicam), comparando las diferentes presentaciones comerciales dispensadas en julio de 2015 con las de julio de 2017.

Esta investigación utilizó datos administrativos ya disponibles, procesados de tal manera que resulta imposible determinar la identidad de los individuos participantes, por lo que no requirió aprobación de un Comité de Ética de Investigación ni tuvo que obtener consentimiento informado; corresponde al caso de datos inidentificables que describe el Art. 11.3.e) de la Ley 25.326, de Protección de los Datos Personales.

\section{RESULTADOS}

La lista de fármacos $\mathrm{A}$, excluidos de la cobertura al $100 \%$, comprende ocho SySADOA, como monodroga o en combinaciones a dosis fijas, mientras que la lista de fármacos $B$ incluye los 15 productos del capítulo M01A de la clasificación ATC -no afectados por la medida- y el paracetamol (Tabla 1).

Tabla 1. Fármacos del capítulo M01A de la clasificación $\mathrm{ATC}^{*}$, según condición de cobertura luego de la Resolución 439/2016. Argentina, 2016.

$\begin{array}{ll}\text { Excluidos del subsidio social } & \begin{array}{l}\text { Incluidos en el subsidio social } \\ \text { (Fármacos A) }\end{array} \\ \text { (Fármacos B) } \\ \text { condroitín,sulfato } & \text { celecoxib } \\ \text { condroitín,sulfato + glucosamina } & \text { clonixinato lisina } \\ \text { diacereína } & \text { dexketoprofeno } \\ \text { diacereína + glucosamina } & \text { diclofenac } \\ \text { diacereína + meloxicam } & \text { diclofenac+asoc } \\ \text { glucosamina } & \text { dipirona } \\ \text { glucosamina + meloxicam } & \text { etoricoxib } \\ \text { insaponificables de palta-soja } & \text { flurbiprofeno } \\ & \text { ibuprofeno } \\ & \text { indometacina } \\ & \text { ketoprofeno } \\ & \text { meloxicam } \\ & \text { naproxeno } \\ & \text { paracetamol } \\ & \text { piroxicam }\end{array}$

Fuente: Elaboración propia con base en datos del Instituto Nacional de Servicios Sociales para Jubilados y Pensionados.

*Capítulo "Productos antiinflamatorios y antirreumáticos, no esteroides" de la clasificación Anatómica-Terapéutica-Química de la Organización Mundial de la Salud ${ }^{(38)}$.

\section{Dispensa antes y después de la intervención}

En el período previo a la intervención se dispensaron, en promedio, 364.000 envases mensuales de fármacos $\mathrm{A}$, que se redujeron a 139.000 en el período posterior, un descenso del $61,6 \%$. La dispensa de fármacos B no se elevó en forma compensatoria, sino que se redujo levemente entre los períodos previo y posterior, pasando de 504.000 a 473.000 envases por mes, respectivamente, con un descenso del 6,1\%. El descenso afectó en forma similar a la lista completa de fármacos A (Tabla 2).

El número de usuarios prevalentes en el período previo fue de 328.400 por mes para

Tabla 2. Dispensa mensual promedio de fármacos A en los períodos previo y posterior a su exclusión del subsidio social. Argentina, 2015-2017.

\begin{tabular}{|lccc}
\hline Fármacos & $\begin{array}{c}\text { Período } \\
\text { previo } \\
01 / 2015 \mathrm{a} \\
03 / 2016 \\
\text { Envases } \\
\text { por mes }\end{array}$ & $\begin{array}{c}\text { Período } \\
\text { posterior } \\
08 / 2016 \mathrm{a}\end{array}$ & $\begin{array}{c}\text { Variación } \\
(\%)\end{array}$ \\
$\begin{array}{lcc}\text { Envases } \\
\text { por mes }\end{array}$ & \\
\hline glucosamina + meloxicam & 180.936 & 71.546 & $-60,5$ \\
\hline condroitín, sulfato + glucosamina & 58.974 & 15.686 & $-73,4$ \\
\hline insaponificables & 50.467 & 25.302 & $-49,9$ \\
\hline glucosamina & 34.583 & 9.577 & $-72,3$ \\
\hline diacereína & 24.728 & 10.510 & $-57,5$ \\
\hline diacereína + glucosamina & 8.722 & 4.132 & $-52,6$ \\
\hline diacereína + meloxicam & 4.937 & 2.616 & $-47,0$ \\
\hline condroitín, sulfato & 214 & 96 & $-55,0$ \\
\hline Total & 363.561 & 139.466 & $-61,6$
\end{tabular}

Fuente: elaboración propia con base en datos del por el Instituto Nacional de Servicios Sociales para Jubilados y Pensionados.

los fármacos A y 382.400 por mes para los $\mathrm{B}$, de los cuales 73.900 consumían fármacos de ambas listas. La prevalencia mensual de uso en el período previo a la medida sobre el total del padrón de beneficiarios fue del $6,7 \%$ para los fármacos $\mathrm{A}$ y del $7,8 \%$ para los $\mathrm{B}$, descendiendo a $2,7 \%$ y a $7,7 \%$, respectivamente, en el período posterior, una reducción del $60 \%$ en los usuarios de la lista A y del $0,4 \%$ en los de la lista B (Figura 1). 


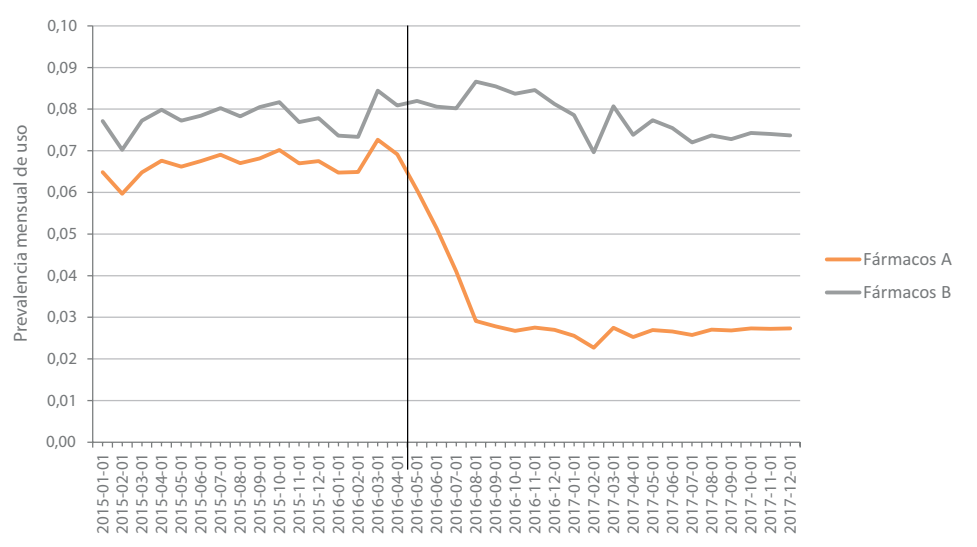

Figura 1. Prevalencia mensual de uso de fármacos para la artrosis excluidos (fármacos $A$ ) y no excluidos (fármacos B) del subsidio social. Argentina, 2015-2017.

Fuente: Elaboración propia con base en datos del Instituto Nacional de Servicios Sociales para Jubilados y Pensionados. Nota: La línea vertical señala la fecha del cambio de cobertura.

El precio total de los fármacos A dispensados siguió una evolución análoga, en un contexto general de inflación, que en el período de tres años estudiado ascendió a $125 \%$. El monto mensual promedio del precio de venta al público de los fármacos $\mathrm{A}$, dispensados en el período previo a la medida, fue de $\$ 145,3$ millones por mes, descendió rápidamente en los siguientes cuatro meses y alcanzó un promedio de $\$ 87,1$ millones en el período posterior, con un descenso del $40,0 \%$ en valor nominal y del $63,4 \%$ en valores constantes de enero de 2015 (Figura 2). Los fármacos B, por su parte, alcanzaron un promedio de $\$ 74,5$ millones por mes en el período previo y ascendieron a $\$ 126,2$ en el posterior, que en valores constantes representa un aumento del 3,6\% entre ambos períodos (Figura 2).

La cobertura promedio sobre el precio de venta al público de los fármacos $\mathrm{A}$ en el

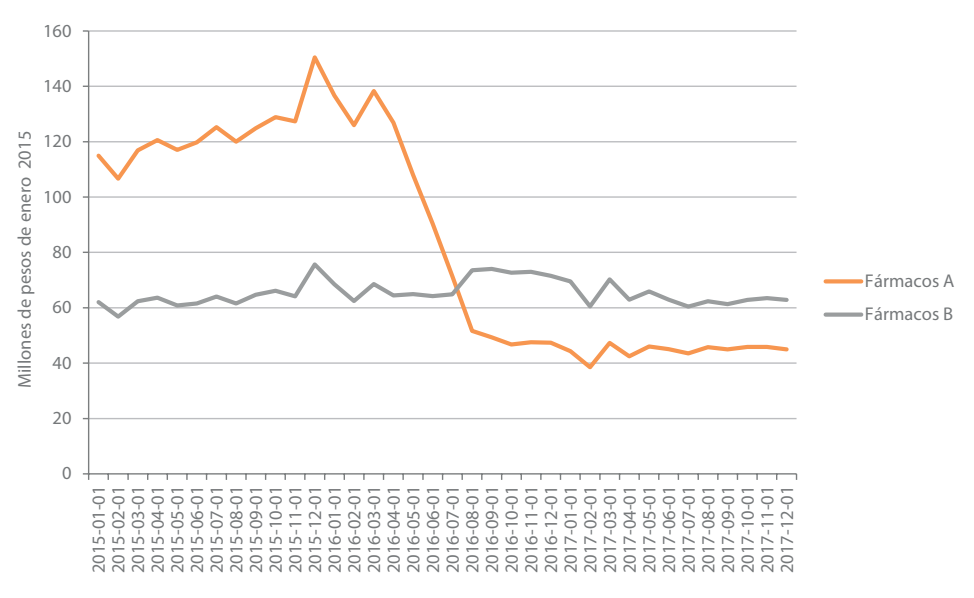

Figura 2. Precio de venta al público del total de fármacos para la artrosis excluidos (fármacos A) y no excluidos (fármacos B) del subsidio social, que se dispensaron mensualmente, medido en pesos de enero de 2015. Argentina, 2015-2017..

Fuente: Elaboración propia con base en datos del Instituto Nacional de Servicios Sociales para Jubilados y Pensionados. Nota: La línea vertical señala la fecha del cambio de cobertura. 


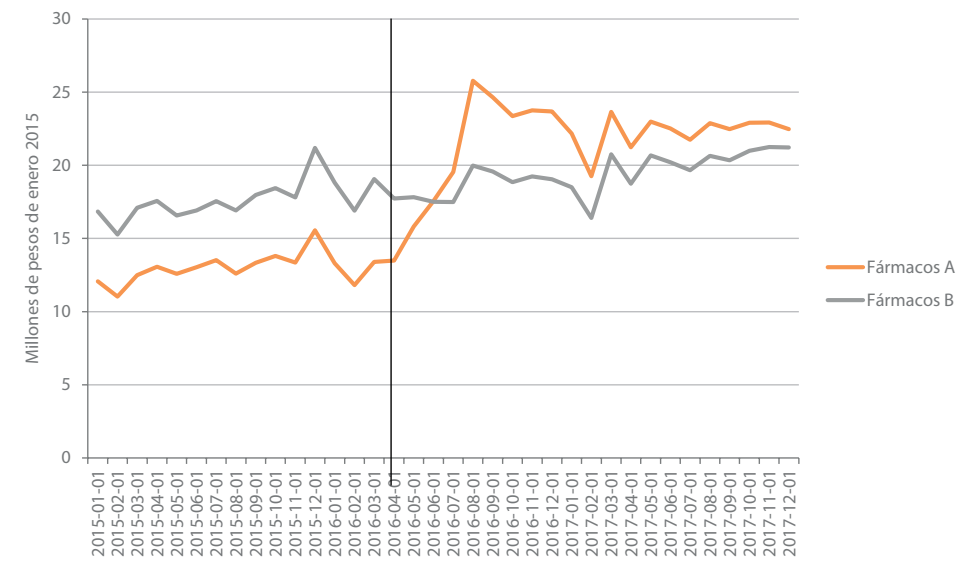

Figura 3. Evolución del gasto de bolsillo de los beneficiarios en fármacos para la artrosis excluidos (fármacos A) o no excluidos (fármacos B) del subsidio social, medido en pesos de enero de 2015. Argentina, 2015-2017.

Fuente: Elaboración propia con base en datos del Instituto Nacional de Servicios Sociales para Jubilados y Pensionados. Nota: La línea vertical señala la fecha del cambio de cobertura.

período previo fue del $89,6 \%$, lo que refleja que el $79,2 \%$ de las dispensas recibían el descuento del $100 \%$ por el subsidio social. $\mathrm{Al}$ excluirse los fármacos $\mathrm{A}$ de este beneficio, la cobertura promedio descendió al 50\% en el período posterior. En consecuencia, el gasto de bolsillo total de los afiliados en fármacos A subió de $\$ 15$ millones por mes en el período previo a $\$ 43,5$ millones por mes en el posterior, lo que significa un aumento del $189 \%$. El gasto de bolsillo en los fárma$\cos B$, por su parte, pasó de $\$ 20,5$ millones por mes a $\$ 35,9$ millones por mes, con un crecimiento del $84,7 \%$. A valores constantes de enero de 2015, el aumento de gasto de bolsillo total fue del $75,8 \%$ para los fármacos A y del 11,9\% para los B (Figura 3 ).

\section{Prescripción inicial y continuidad del tratamiento}

A lo largo de los 36 meses estudiados, los fármacos A se dispensaron a 1.196 .000 personas diferentes, prácticamente 1 de cada 4 beneficiarios del INSSJP. La tasa mensual de incidencia de nuevos tratamientos con fármacos A era de 6 cada 1.000 beneficiarios en el período previo y se redujo a 3 cada 1.000 en el período posterior.

A pesar de tratarse de medicamentos de uso crónico, la continuidad de uso en el período previo a la medida no era muy elevada: solo el $69 \%$ de los pacientes que iniciaban tratamiento con un fármaco $\mathrm{A}$ recibían una segunda dispensa, y solo el $54 \%$, una tercera. En el período posterior estos porcentajes descendieron al $50 \%$ y $33 \%$, respectivamente.

La reducción del $61,6 \%$ en la dispensa de fármacos $\mathrm{A}$, luego de la medida, se debe entonces a una combinación de una caída a la mitad del inicio de nuevos tratamientos y un descenso sustancial de la continuidad de los tratamientos ya iniciados.

El registro de la especialidad del médico prescriptor se estaba implementando durante el período de estudio y no llegó a completarse: había datos del $40 \%$ de los prescriptores en enero de 2015 y alcanzó un $75 \%$ desde julio de 2017. Teniendo en cuenta estas limitaciones, se encontró que al menos el $80 \%$ de los nuevos tratamientos con fármacos $\mathrm{A}$ son indicados por los médicos de cabecera, y solo un $20 \%$ por los especialistas. 


\section{Dispensa según sexo y unidades de gestión local}

La prevalencia de uso mensual de las mujeres beneficiarias duplicó a la de los varones, tanto antes $(8,9 \%$ y $4,4 \%$, respectivamente, en julio de 2015) como después de la medida (3,2\% y $1,6 \%$, respectivamente, en julio de 2017 ).

Las 38 unidades de gestión local tuvieron un amplio rango de prevalencia de uso antes y después de la medida, observándose también tendencias regionales. En julio de 2015 se registró el uso mínimo en la Ciudad Autónoma de Buenos Aires $(3,6 \%)$ y el máximo en las provincias de Catamarca, La Rioja y Tucumán (todas en 12,8\%), con una relación de 3,5 a 1. En julio de 2017, el mínimo se dio nuevamente en la Ciudad Autónoma de Buenos Aires $(1,6 \%)$ y el máximo en La Rioja $(4,7 \%)$ (Tabla 3$)$

Tabla 3. Prevalencia de uso de los fármacos $A$ antes y después de la medida, según la unidad de gestión local de residencia del beneficiario. Argentina, 2015 a 2017.

\begin{tabular}{|c|c|c|c|}
\hline Unidad de gestión local & $\begin{array}{c}07 / 2015 \\
(\%)\end{array}$ & $\begin{array}{c}07 / 2017 \\
(\%)\end{array}$ & $\begin{array}{c}\text { Descenso } \\
(\%)\end{array}$ \\
\hline La Rioja & 12,8 & 4,7 & 63,3 \\
\hline Catamarca & 12,8 & 4,2 & 67,2 \\
\hline Tucumán & 12,8 & 3,7 & 71,1 \\
\hline San Juan & 12,3 & 2,5 & 79,7 \\
\hline Mendoza & 11,3 & 3,6 & 68,1 \\
\hline Salta & 11,2 & 3,6 & 67,9 \\
\hline Río Cuarto & 10,0 & 3,2 & 68,0 \\
\hline Entre Ríos & 10,0 & 3,7 & 63,0 \\
\hline San Luis & 9,8 & 2,4 & 75,5 \\
\hline Jujuy & 9,4 & 3,6 & 61,7 \\
\hline Córdoba & 8,5 & 2,9 & 65,9 \\
\hline Formosa & 8,5 & 2,3 & 72,9 \\
\hline Concordia & 8,5 & 3,2 & 62,4 \\
\hline Santiago del Estero & 8,5 & 2,8 & 67,1 \\
\hline Rio Negro & 8,4 & 3,1 & 63,1 \\
\hline Chaco & 8,2 & 3,2 & 61,0 \\
\hline Rosario & 7,7 & 2,9 & 62,3 \\
\hline Mar del Plata & 7,4 & 2,2 & 70,3 \\
\hline Total del País & 7,2 & 2,6 & 63,9 \\
\hline Neuquén & 7,1 & 2,4 & 66,2 \\
\hline Bahía Blanca & 7,1 & 2,7 & 62,0 \\
\hline Misiones & 7,1 & 2,5 & 64,8 \\
\hline Santa Fe & 6,7 & 3,0 & 55,2 \\
\hline Morón & 6,5 & 2,6 & 60,0 \\
\hline Corrientes & 6,5 & 2,7 & 58,5 \\
\hline Quilmes & 6,3 & 2,6 & 58,7 \\
\hline Junín & 6,0 & 2,1 & 65,0 \\
\hline Chivilcoy & 6,0 & 2,4 & 60,0 \\
\hline Luján & 5,9 & 2,3 & 61,0 \\
\hline San Justo & 5,7 & 2,3 & 59,6 \\
\hline Lanús & 5,6 & 2,2 & 60,7 \\
\hline La Pampa & 5,5 & 2,7 & 50,9 \\
\hline San Martin & 4,9 & 2,1 & 57,1 \\
\hline La Plata & 4,9 & 1,8 & 63,3 \\
\hline Azul & 4,9 & 2,3 & 53,1 \\
\hline Chubut & 4,6 & 2,7 & 41,3 \\
\hline Tierra del Fuego & 4,4 & 1,9 & 56,8 \\
\hline Santa Cruz & 4,2 & 2,6 & 38,1 \\
\hline Ciudad Autónoma de Buenos Aires & 3,6 & 1,6 & 55,6 \\
\hline
\end{tabular}

Fuente: Elaboración propia con base en datos del Instituto Nacional de Servicios Sociales para Jubilados y Pensionados. 


\section{Selección del producto comercial}

La combinación de glucosamina y meloxicam fue el SySADOA más prescrito antes de la medida, con el $49,8 \%$ de los envases dispensados. Se evaluó la selección de marcas comerciales en función del precio, utilizando como referencia la presentación de 30 sobres de granulado con 1.500 mg de glucosamina y 15 mg de meloxicam. En julio de 2015, había en el mercado 13 alternativas comerciales, con un precio de venta al público promedio de \$390 (rango: \$274-\$451), y el promedio del precio de venta al público de los productos efectivamente dispensados fue de $\$ 417$, un $92 \%$ del precio máximo, debido a que la marca más cara fue también la más utilizada (43\% de los envases). En julio de 2017, había 15 presentaciones con precio promedio de \$689 (rango: \$279 - \$808), no había variado la selección de marcas, y el precio promedio de lo dispensado fue de $\$ 745$, nuevamente un $92 \%$ del precio máximo.

\section{El caso del meloxicam}

El antiinflamatorio no esteroide meloxicam se comercializa en combinaciones a dosis fija con glucosamina y con diacereína, ambas incluidas en la lista de fármacos $\mathrm{A}, \mathrm{y}$ también como monodroga, incluida en la lista de fármacos B. En el período previo a la intervención representaban el $79 \%$ y el $21 \%$, respectivamente, de los envases de medicamentos con meloxicam dispensados. En el período posterior, a pesar de un descenso del $60 \%$ en la dispensa de las combinaciones de SySADOA con meloxicam, no se observó un aumento compensatorio de su dispensa como monodroga, aunque esta última seguía siendo aceptada para la cobertura al 100\%.

\section{DISCUSIÓN}

Al excluir a los SySADOA de los medicamentos que pueden ser cubiertos al $100 \%$ por el INSSJP, aunque conservando la cobertura del $50 \%$, se produjo una caída del $61,6 \%$ en el número mensual de envases dispensados, junto con un descenso del $63,4 \%$ en el monto total del precio de venta al público, a valores constantes de enero de 2015. Por la reducción del porcentaje de cobertura y a pesar del importante descenso de las cantidades dispensadas, el gasto de bolsillo de los afiliados aumentó un $75,8 \%$ a valores constantes. Todos estos efectos se completaron durante un período inicial de cuatro meses, y se mantuvieron estables durante los 16 meses posteriores.

Llama la atención que al excluirse de la cobertura al 100\% la combinación de glucosamina con meloxicam, que representaba la mitad de los envases de SySADOA dispensados antes de la medida, no se haya reorientado la prescripción hacia el meloxicam como monodroga, que mantenía la cobertura al $100 \%$. Esto podría reflejar la falta de información del médico y del paciente sobre los alcances de la nueva normativa, o bien cierta "inercia organizacional" que demora la adaptación de las prácticas a los cambios de las reglas institucionales. Tampoco se produjo un desplazamiento de la dispensa hacia las presentaciones comerciales de menor precio, a pesar de que esto hubiera generado un ahorro sustancial para el beneficiario.

Las combinaciones de un SySADOA con un AINE, que representaron en este estudio casi el $50 \%$ de las dispensas, resultan poco racionales, ya que reúnen un analgésico de efecto rápido, a utilizar por el menor tiempo posible, con un fármaco de acción lenta cuyos beneficios se esperan luego de varios meses de tratamiento. Aunque podría argumentarse que la combinación es útil en el momento inicial del tratamiento y debería ser sustituida con el SySADOA como monodroga luego de unas pocas semanas, en la práctica se convierte en el tratamiento crónico de la artrosis, con una exposición prolongada a los efectos adversos del AINE. En base a estas y otras consideraciones, la combinación de glucosamina y meloxicam fue retirada de la comercialización en 2020 por la Administración Nacional de Medicamentos, Alimentos y Tecnología Médica ${ }^{(42)}$.

Se ha señalado más arriba que en las intervenciones de desinversión es preciso medir 
la eventual reorientación del consumo hacia otros medicamentos no afectados por la medida. En este trabajo no se observó un aumento de la dispensa de los fármacos alternativos -AINEs y paracetamol- a partir de la medida, aunque estos conservaban la posibilidad de ser cubiertos al 100\%. Debe destacarse que el sistema de información utilizado no captura la dispensa de medicamentos de venta libre, la que podría haberse modificado.

Este estudio no analizó resultados de salud. Sin embargo, a la luz de la cuestionada eficacia de los SySADOA, no se esperan consecuencias importantes de la reducción de su dispensa. Es destacable que casi la mitad de los SYSADOA dispensados eran combinaciones con meloxicam, que exponen a esta población vulnerable por su edad avanzada al uso prolongado de un AINE, por lo que puede esperarse que el resultado obtenido reduzca el riesgo de sus efectos adversos digestivos y cardiovasculares ${ }^{(43,44)}$.

Para el financiador, el resultado económico fue una reducción sustancial del gasto en SYSADOA. Por otra parte, el aumento en el gasto de bolsillo de los afiliados resultó un efecto no deseado, ya que implica la asignación de estos recursos siempre escasos a fármacos de bajo valor terapéutico potencial $^{(45)}$. No hubo evidencia de que prestadores y pacientes recurrieran a dos estrategias disponibles para limitar el gasto de bolsillo: reorientar el tratamiento farmacológico a productos que conservaban la cobertura al $100 \%$ y, para aquellos afiliados que mantuvieron el tratamiento con SYSADOA, promover la selección de marcas comerciales con menor precio. Estas estrategias podrían haberse abordado con una comunicación previa a prescriptores y pacientes que incluyera la descripción de la medida, sus fundamentos y las alternativas disponibles, así como, eventualmente, una introducción gradual de la normativa que facilitara la adaptación. Aunque no se realizó una evaluación formal de la satisfacción de pacientes y prestadores frente a la medida, el aumento del gasto de bolsillo de los afiliados y la falta de adaptación de los prescriptores a las nuevas medidas se asocian presumiblemente con insatisfacción.
La medida que se analiza tiene también consecuencias en el mercado farmacéutico, ya que el INSSJP es el principal comprador de medicamentos en Argentina, estimándose que alcanza entre el $35 \%$ y el $40 \%$ del mercado total ${ }^{(15)}$. Por este motivo, sus políticas de medicamentos influyen en el perfil de la oferta de productos y de la fijación de precios para el conjunto de la sociedad. Dado que los SYSADOA son fármacos de uso preferente en adultos mayores y que el $76 \%$ de la población argentina mayor de 65 años está afiliada al INSSJP(17), la reducción observada del $61,6 \%$ en la dispensa como consecuencia de una limitación del nivel de cobertura tiene implicancias mayores en ese segmento del mercado. Generalizando este efecto, una política de cobertura de medicamentos por parte del INSSJP que se focalice en fármacos de eficacia probada tiene el potencial de mejorar la racionalidad de todo el mercado de medicamentos en Argentina.

Como limitaciones de este trabajo debe señalarse la ausencia de un grupo paralelo de control, ya que la intervención de reducción de la cobertura que se describe se aplicó simultáneamente a toda la población de beneficiarios. En segundo lugar, el estudio de la eventual reorientación del consumo de fármacos se limitó al grupo de los AINE, cuando otros grupos farmacológicos -como los corticosteroides o los analgésicos opiáceostambién podrían haberse visto afectados. Además, como ya se señaló, la investigación no abordó el componente de satisfacción de beneficiarios y prestadores, ni el impacto sobre la salud.

Las estrategias de desinversión representan un desafío para las instituciones financiadoras de la atención de la salud, en un esfuerzo por conciliar la mejor asignación posible de los recursos presupuestarios con la mejoría de los resultados de salud y la satisfacción de pacientes y prestadores. Estas intervenciones enfrentan múltiples resistencias de todas las partes interesadas, que requieren prever sus efectos sobre los diversos aspectos ya mencionados, con un compromiso entre lo ideal y lo factible, lo que Mac Kean et al resumen como "el arte de lo posible" ${ }^{\prime 27,28)}$. 
Para mejorar las chances de éxito, el proceso debe ser transparente, las medidas deben estar bien fundamentadas y haber sido consensuadas previamente con los actores clave ${ }^{(28,46)}$.

\section{REFERENCIAS BIBLIOGRÁFICAS}

1. Información Farmacoterapéutica. Tratamiento de la artrosis. INFAC [Internet]. 2018;26(1):1-7 [citado 11 dic 2019]. Disponible en: https://tinyurl.com/2v3678e5.

2. Alfabeta.net. Manual Farmacéutico Online. [Internet]. 2018 [citado 3 nov 2018]. Disponible en: http://www. alfabeta.net

3. Calvo Pita C. Fármacos sintomáticos de acción lenta y administración oral para la artrosis: dudosa eficacia en el control sintomático y nula actividad condroprotectora. El Comprimido [Internet]. 2020;(18):1-7 [citado 10 may 2020]. Disponible en: https://tinyurl.com/3z2thf4v.

4. Gutiérrez-Ibarluzea I, Ibargoyen-Roteta N, BenguriaArrate G, Rada D, Mateos M, Regidor I, Domingo C, González R, Galnares-Cordero L. Sysadoas: Condroprotectores en el tratamiento de la artrosis [Internet]. San Sebastián: Eusko Jaurlaritzaren Argitalpen Zerbitzu Nagusia, Servicio Central de Publicaciones del Gobierno Vasco; 2014 [citado 10 ene 2019]. Disponible en: https://tinyurl.com/x2ncs639.

5. Fidelix TS, Macedo CR, Maxwell LJ, Fernandes Moça Trevisani V. Diacerein for osteoarthritis. Cochrane Database of Systematic Reviews. 2014;(2):CD005117. doi: 10.1002/14651858.CD005117.pub3.

6. Singh JA, Noorbaloochi S, MacDonald R, Maxwell LJ. Chondroitin for osteoarthritis. Cochrane Database of Systematic Reviews. 2015;(1):CD005614. doi: 10.1002/14651858.CD005614.pub2.

7. Hochberg MC, Martel-Pelletier J, Monfort J, Möller I, Castillo JR, Arden N, et al. Combined chondroitin sulfate and glucosamine for painful knee osteoarthritis: a multicentre, randomised, double-blind, non-inferiority trial versus celecoxib. Annals of the Rheumatic Diseases. 2016;75(1):37-44. doi: 10.1136/annrheumdis-2014-206792.

8. Fransen M, Agaliotis M, Nairn L, Votrubec M, Bridgett L, Su S, et al. Glucosamine and chondroitin for knee ostearthritis: a double-blind randomized placebo-controlled clinical trial evaluating single and combination regimens. Annals of the Rheumatic Diseases. 2015;74(5):851-858. doi: 10.1136/annrheumdis-2013-203954.

9. Roman-Blas JA, Castañeda S, Sánchez-Pernaute O, Largo R, Herrero-Beaumont G. Combined treatment with chondroitin sulfate and glucosamine sulfate shows no superiority over placebo for reduction of joint pain and functional impairment in patients with knee osteoarthritis: A six-month multicenter, randomized, double-blind, placebo-controlled clinical trial. Arthritis \& Rheumatology. 2017;69(1):77-85. doi: 10.1002/art.39819.

10. Reginster JY, Dudler J, Blicharski T, Pavelka K. Pharmaceutical-grade chondroitin sulfate is as effective as celecoxib and superior to placebo in symptomatic knee osteoarthritis: The ChONdroitin versus CElecoxib versus Placebo Trial (CONCEPT). Annals of the Rheumatic Diseases. 2017;76(9):1537-1543. doi: 10.1136/ annrheumdis-2016-210860.

11. Haute Autorité de Santé. Art 50, Zondar, Chondrosulf, Piasclédine, Dolenio, Flexea, Osaflexan, Structoflex et Voltaflex: service médical rendu insuffisant dans le traitement symptomatique de l'arthrose [Internet] HAS, 2013 [citado 20 jun 2020]. Disponible en: https:// tinyurl.com/36u2jy73.

12. Ministerio de Salud. Resolución 310/2004 [Internet]. 2004 [citado 10 mar 2020]. Disponible en: https:// tinyurl.com/2nwddmf3.

13. Instituto Nacional de Servicios Sociales para Jubilados y Pensionados. Historia [Internet]. 2019 [citado 4 feb 2019]. Disponible en: https://www.pami.org.ar/ historia

14. Instituto Nacional de Servicios Sociales para Jubilados y Pensionados. Listado de precios de medicamentos para afiliados [Internet]. 2019 [citado 4 feb 2019]. Disponible en: https://tinyurl.com/a4absch8.

15. Bisang R, Luzuriaga JP, San Martín M. El mercado de los medicamentos en Argentina. Fundación CECE [Internet]. 2017 [citado 10 jun 2020]. Disponible en: https://tinyurl.com/2d2jykz7.

16. Instituto Nacional de Servicios Sociales para Jubilados y Pensionados. Resolución 337/2005. Boletín del Instituto [Internet]. 2005;1(96):1-19 [citado 10 may 2020]. Disponible en: https://tinyurl.com/r3nd8yut.

17. Instituto Nacional de Servicios Sociales para Jubilados y Pensionados. El Modelo prestacional y los desafíos del PAMI [Internet]. Consejo Profesional de Ciencias Económicas de la Ciudad Autónoma de Buenos Aires; 2017 [citado 20 jun 2020]. Disponible en: https:// tinyurl.com/9y9upyjh.

18. Cermignani EC, Cañás M. Medicamentos esenciales en la Atención Primaria de la Salud. En: Roa R, Torres R. (eds). Atención Primaria de la Salud. En prensa, 2020. 
19. Argentina, Poder Ejecutivo Nacional. Decreto 150/92: Normas para el registro, elaboración, fraccionamiento, prescripción, expendio, comercialización, exportación e importación de medicamentos [Internet] 1992 [citado 15 dic 2020]. https://tinyurl.com/f7hdskxz.

20. Cañás M, Buschiazzo HO, Urtasun MA. Valor terapéutico y precio de los nuevos fármacos comercializados en Argentina: ¿valen lo que cuestan? Salud Colectiva. 2019;15:e1962. doi: 10.18294/sc.2019.1962.

21. Cañás $M$, Carlson $S$, Petinelli $A$, Raimondi $M$, et al. Medicamentos de riesgo inaceptable comercializados en 7 países de América latina. Researchgate. doi: 10.13140/ RG.2.2.11872.48645.

22. Trionfetti $M$, Mordujovich-Buschiazzo $P$, Cañás $M$ Marín G, Buschiazzo HO, Marín L, et al. Medicamentos presentes en el mercado farmacéutico argentino y retirados de otros mercados internacionales por efectos adversos graves. En: Resúmenes de la XXVII Reunión de Gapurmed. La Plata: Comisión de Investigaciones Científicas de la Provincia de Buenos Aires; 2019.

23. Giulietti M, Carlson S, Cañás $M$, Buschiazzo H, M. de Buschiazzo P. Medicamentos en combinaciones a dosis fijas en el mercado argentino durante el año 2006. 1ra etapa: AINES. Póster XIV Reunión del DURG-La, Foro-Taller Internacional: "Acceso Universal a los Medicamentos Antiretrovirales: Encuentro Universitario de Farmacoterapéutica". Santo Domingo, República Dominicana 26 al 29 de Septiembre de 2007.

24. Cañás M, Wirtz V, Ibáñez SE, Vargas A, Melgarejo $S$, Valsecia M. Estudio de utilización de medicamentos de combinaciones a dosis fijas (CDF): disponibilidad comercial y riesgo beneficio en 4 países latinoamericanos. En: Resúmenes XX Reunión anual GAPURMED 2011, San Luis, Argentina.

25. Instituto Nacional de Servicios Sociales para Jubilados y Pensionados. Resolución 439/2016. Boletín del Instituto. 2016;12(2516):8-11.

26. Repullo JR. Taxonomía práctica de la «desinversión sanitaria» en lo que no añade valor, para hacer sostenible el Sistema Nacional de Salud. Revista de Calidad Asistencial. 2012;27(3):130-138. doi: 10.1016/j. cali.2012.02.010.

27. MacKean G, Noseworthy T, Elshaug AG, Leggett L, Littlejohns P, Berezanski J, et al. Health technology reassessment: the art of the possible. International Journal of Technology Assessment in Health Care. 2013;29(4):418 423. doi: $10.1017 / \mathrm{S} 0266462313000494$.

28. Parkinson B, Sermet C, Clement F, Crausaz S, Godman B, Garner S, et al. Disinvestment and value-based purchasing strategies for pharmaceuticals: an international review. Pharmacoeconomics. 2015;33(9):905-924. doi: 10.1007/s40273-015-0293-8.

29. Pichetti S, Sorasith C, Sermet C. Analysis of the impact of removing mucolytics and expectorants from the list of reimbursable drugs on prescription rates: A time-series analysis for France 1998-2010. Health Policy. 2011;102(23):159-169. doi: 10.1016/j.healthpol.2011.07.001.
30. Antoñanzas Villar F, Rodríguez-lbeas R, JuárezCastelló CA, Lorente Antoñanzas MR. Impacto del Real Decreto-Ley 16/2012 sobre el copago farmacéutico en el número de recetas y en el gasto farmacéutico. Revista Española de Salud Pública. 2014;88(2):233-249. doi: 10.4321/S1135-57272014000200006.

31. Compairé Bergua I, Compairé Bergua A, Arner Navarro JA, García Lerma D, Gazo AR. Análisis de las consecuencias de la desfinanciación de medicamentos del 1 de septiembre de 2012. Farmacéuticos Comunitarios 2014;6(2):5-10

32. Pichetti S, Sermet $C$. Le déremboursement des médicaments en France entre 2002 et 2011: éléments d'évaluation. Questions d'économie de la santé. 2011;(167):1-7.

33. Lasio L. Delisting of pharmaceuticals from insurance coverage: effects on consumption, pricing and expenditures in France [Internet]. 2016. [citado 9 nov 2019]. Disponible en: https://tinyurl.com/4zwxz6bf.

34. Kenneally M, Walshe V. Pharmaceutical cost-containment policies and sustainability: recent Irish experience. Value in Health. 2012;15:389-393. doi:10.1016/j. jval.2011.10.007.

35. Gür Ali O, Topaler B. How removing prescription drugs from reimbursement lists increases the pharmaceutical expenditures for alternatives. European Journal of Health Economics. 2011;12:553-562. doi 10.1007/ s10198-010-0270-2.

36. Chambers JD, Rane PB, Neumann PJ. The impact of formulary drug exclusion policies on patients and healthcare costs. American Journal of Managed Care. 2016;22(8):524-531.

37. Park Y, Raza S, George A, Agrawal R, Ko J. The effect of formulary restrictions on patient and payer outcomes: a systematic literature review. Journal of Managed Care \& Specialty Pharmacy. 2017;23(8):893-901. doi: 10.18553/jmcp.2017.23.8.893.

38. WHO Collaborating Centre for Drug Statistics Methodology. ATC index with DDDs [Internet]. 2020 [citado 20 feb 2020]. Disponible en: https://tinyurl.com/ ys5zn5yr.

39. Cavallo A, Bertolotto M. Serie completa de inflación de Argentina desde 1943 a 2016 [Internet]. 2016 [citado 20 feb 2020]. Disponible en: http://dx.doi.org/10.2139/ ssrn. 2787276

40. Dirección General de Estadística y Censos. Índice de precios al consumidor [Internet]. 2019 [citado 10 ene 2019]. Disponible en: https://tinyurl.com/wes75mcu.

41. Hallas J, Støvring $H$, Pottegård A. Individual-level drug utilization analyses. En: Elseviers $M$, Wettermark B, Almarsdóttir AB, (eds). Drug Utilization Research: Methods and Applications. New York: John Wiley \& Sons; 2016. p. 68-76.

42. Administración Nacional de Medicamentos, Alimentos y Tecnología Médica. Disposición 528/2020. Boletín Oficial de la República Argentina [Internet]. 7 feb 
2020 [citado 10 mar 2020]. Disponible en: https://tinyurl. com/23v857wj.

43. Wolfe MM, Lichtenstein DR, Singh G. Gastrointestinal toxicity of nonsteroidal antiinflammatory drugs. New England Journal of Medicine. 1999;340(24):18881899. doi: 10.1056/NEJM199906173402407.

44. Prozzi GR, Cañás M, Urtasun MA, Buschiazzo HO, Dorati CM, Mordujovich-Buschiazzo P. Riesgo Cardiovascular de los antiinflamatorios no esteroideos. Medicina (Buenos Aires). 2018;78(5):349-355.
45. Capellà D, Laporte JR. Métodos aplicados en estudios descriptivos de utilización de medicamentos. En: Laporte JR, Tognoni G. Principios de epidemiología del medicamento. Barcelona: Salvat; 1983.

46. Pace J, Laba TL, Nisingizwe MP, Lipworth W. Formulating an ethics of pharmaceutical disinvestment. Journal of Bioethical Inquiry. 2020;17(1):75-86. doi: 10.1007/s11673-020-09964-Z.

\section{FORMA DE CITAR}

Urtasun MA, Noble M, Cañás M, Bustin, J, Mastai RC, Regueiro AJ. Reducción de la cobertura social para los fármacos antiartrósicos sintomáticos de acción lenta: una iniciativa de desinversión en Argentina, 2015-2017. Salud Colectiva. 2021;17:e3246. doi: 10.18294/sc.2021.3246.

Recibido: 22 oct 2020 | Versión final: 17 dic 2020 | Aprobado: 26 dic 2020 | Publicado en línea: 6 mar 2021

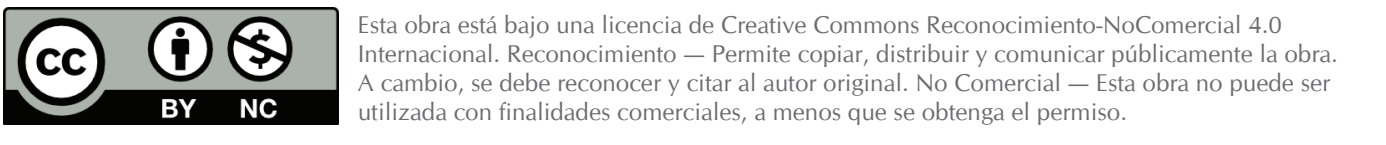

https://doi.org/10.18294/sc.2021.3246 